Research Article

\title{
Research on Dynamic Weapon Target Assignment Based on Cross-Entropy
}

\author{
Lei Hu (D), GuoXing Yi (iD, Chao Huang, Yi Nan, and ZeYuan Xu \\ School of Astronautics, Harbin Institute of Technology, Harbin, Heilongjiang, China \\ Correspondence should be addressed to GuoXing Yi; ygx@hit.edu.cn
}

Received 4 October 2019; Revised 7 December 2019; Accepted 25 January 2020; Published 2 September 2020

Academic Editor: Gareth A. Vio

Copyright $\odot 2020$ Lei Hu et al. This is an open access article distributed under the Creative Commons Attribution License, which permits unrestricted use, distribution, and reproduction in any medium, provided the original work is properly cited.

\begin{abstract}
The weapon target assignment (WTA) is a classical problem of defense-related applications which is proved to be a NP-complete problem. In this paper, a practical and available dynamic weapon target assignment (DWTA) formulation is given which incorporates two meaningful and conflicting objectives, that is, minimizing weapon costs and maximizing combat benefits. As we know, heuristic methods have some shortcomings such as slow convergence speed and local optimum in solving the nonlinear integer optimization problem. To this end, a novel DWTA algorithm based on cross-entropy (CE) method is introduced, where the resources requirement condition for targets is taken into consideration. The CE method associates an estimation problem with the DWTA optimization problem, and then, the estimation problem is transformed into a convex optimization problem. The Karush-Kuhn-Tucker conditions are applied to solve the convex optimization problem, and the iteration formulas to find the optimal solution are deducted. Furthermore, in order to verify the performance of CE method in dealing with the DWTA problem, several simulations in different combat scenarios are implemented. The results reveal that, compared with the benchmark heuristic and Monte-Carlo (MC) methods, there are some notable advantages in solving the DWTA problem based on $\mathrm{CE}$ method with regard to the solution quality and time consumption.
\end{abstract}

\section{Introduction}

Weapon target assignment (WTA) problem is the core content in the research of combat command aided decisionmaking, which can be categorized as a combinatorial optimization problem and nondeterministic polynomial complete problem [1], whose solution space expands exponentially with the increasing of the number of weapons and targets. Generally, WTA problem can be divided into static weapon target assignment (SWTA) problem $[2,3]$ and dynamic weapon target assignment (DWTA) problem [4-6]. In SWTA, all weapons are assigned to targets simultaneously and all information is known; however, in DWTA, many dynamic changes such as time window and weapon consumption should be considered; in that case, the solving algorithm for DWTA problem must have the good real-time performance. Furthermore, some researchers pay much attention to sensor weapon target assignment problem [7-9], where small and sensitive sensors loaded by weapons play a critical role to enhance the combat effectiveness, and in order to fit the combat scene pertinently, multiobjective optimization problem $[10,11]$ is researched to depict the combat purpose in different perspectives.

Concretely, Kline et al. [2] proposed a nonlinear branch and bound algorithm to solve the SWTA problem which sought to minimize the residual value of each target. Zhou et al. [3] proposed a discrete particle swarm optimization (PSO) algorithm to maximize the ratio of global operation effectiveness to the costs of consumed weapons. Lai and $\mathrm{Wu}$ [4] proposed an improved simplified swarm optimization method with two novel schemes to minimize the threat value in the multistage WTA. Chang et al. [5] proposed an artificial bee colony algorithm with heuristic factor initialization to get the minimal expected value of surviving targets for all stages. Mu et al. [7] built a sensor WTA model with the probability of detection and killing, and then multiscale quantum harmonic oscillator algorithm was applied to solve the optimization problem, and Chen et al. [8] used the 
particle swarm optimization algorithm to solve the sensor WTA problem while Xin et al. [9] proposed a marginalreturn-based constructive heuristic method to deal with and solve sensor WTA problem. Juan et al. [12] built a multiobjective WTA formulation and NSGAII algorithm was applied to solve the problem, and Li et al. [10] compared the adaptive NSGAII algorithm and adaptive MOEA/D algorithm and gave a comparison study. Gao et al. [11] proposed the D-NSGA-III-A algorithm with the adaptive operator selection mechanism. Both NSGAII and D-NSGA-III-A are extensions of the GA algorithm.

With the development of computer performance, numerous methods are developed to solve the WTA problems, such as branch and bound [2], PSO [3, 8, 13], artificial bee colony algorithm [5], genetic algorithm [10-12], large-scale neighborhood algorithm [14, 15], and ant colony algorithm $[16,17]$. Some new methods are also applied to deal with the WTA problem such as simplified swarm optimization [4], multiscale quantum harmonic oscillator algorithm [7], marginal-return-based constructive heuristic [9], and Markov decision model [18]. Some scholars are also interested in hybrid algorithms [19-21]. Although the application of these methods provides a variety of ideas for solving WTA problems, it is necessary to further expand the optimization method for the realistic and dynamic combat scenarios. Rubinstein [22] indicated that it is feasible and effective to utilize CE method to estimate the probability of rare event and solve complicated nonlinear combination optimization problems. As a simple but powerful tool, CE method is available for numerous practical applications, such as combination optimization problems [23], deep learning [24], and multi-UAVs task allocation [25, 26].

As an efficient deterministic optimization method, branch and bound method is involved to solve nonlinear optimization problem $[2,25]$. Le Thi et al. [25] compared the performance of CE method and branch and bound method in terms of solving the nonlinear UAV task assignment problem. To evaluate the effectiveness of CE method, Huang et al. [26] applied it in various test problems and compared it with the exhaust search method. The comparable research, among CE method and other benchmark heuristic methods, is insufficient; in this paper, in order to verify the performance of solving the DWTA problem based on CE method, two benchmark heuristic algorithms (PSO and GA) and Monte-Carlo algorithm are compared with the CE method.

The aim of this paper is to reveal the detail process of applying cross-entropy to solve the discrete nonlinear DWTA problem under the multiple resources requirement condition. The contribution of this paper is as follows:

(i) A novel DWTA formulation with two significant and conflicting objectives is constructed, which minimize the weapon costs and maximize the combat benefits. Multiple resources requirement conditions changeable are taken into consideration which limit the feasibility of weapon in multistage combat. The kill probability is randomly selected between zero and one which is distinct from those models with an assumption that all firepower units have the same kill probability for different types of targets [15]. With these considerations, the DWTA formulation given in this paper is more practical and realistic.

(ii) The idea of estimating rare event probability with $\mathrm{CE}$ is extended to the solution of discrete nonlinear DWTA problem. To be specific, the DWTA problem depicted in this paper is transformed into an estimation problem, and then it is subsequently transformed into a convex optimization problem, detailed iteration formulas are deducted in the following text, and then the algorithm of solving DWTA problem based on CE method is acquired.

(iii) 24 DWTA problems of different scales are simulated to cover the different combat scenarios; furthermore, the benchmark heuristic and Monte-Carlo methods are implemented to verify the performance of CE method.

The rest of the paper is structured as follows: in Section 2, a DWTA formulation based on weapon costs and combat benefits under multiple resources requirement condition is presented. Furthermore, in Section 3 we apply CE method to solve the DWTA problem and give the elaborate deduction procedure based on Lagrange multiplier method and KKT conditions. Section 4 employs several combat scenarios to verify the performance of $\mathrm{CE}$ method compared with benchmark heuristic and MC methods. Section 5 summarizes the research contents of this paper and prospects the follow-up research work.

\section{DWTA Formulation}

2.1. Combat Scenario. The purpose of WTA problem is to get maximal combat benefits by utilizing weapons to intercept targets under certain or uncertain combat environment. In this paper, we present a DWTA formulation based on consumed weapon costs and combat benefits incorporating multiple resources requirement condition, and a typical scenario of the weapons against the targets is shown in Figure 1 . We assume that there are $n$ targets and $m$ types of weapon, and each target has different combat ability value and resources requirement in the combat scenario; at the same time, the resources contained by each weapon and the inventory quantity of each weapon are taken into consideration. The solution of the DWTA problem is based on the following assumptions.

Assumption 1. The kill probability of using the same weapon to intercept different target is different and the kill probability of using different weapon to intercept the same target is different. The value of kill probability is randomly selected.

Assumption 2. The weapon satisfied with the resources requirement condition of target can be used to interception task. As depicted in Figure 1, weapon 1 can intercept target 1; however, weapon 2, which does not satisfy the resources condition of target 1 , is limited to intercept target 1.

Assumption 3. Every type of weapon can be launched once simultaneously, so the objective of WTA is to 


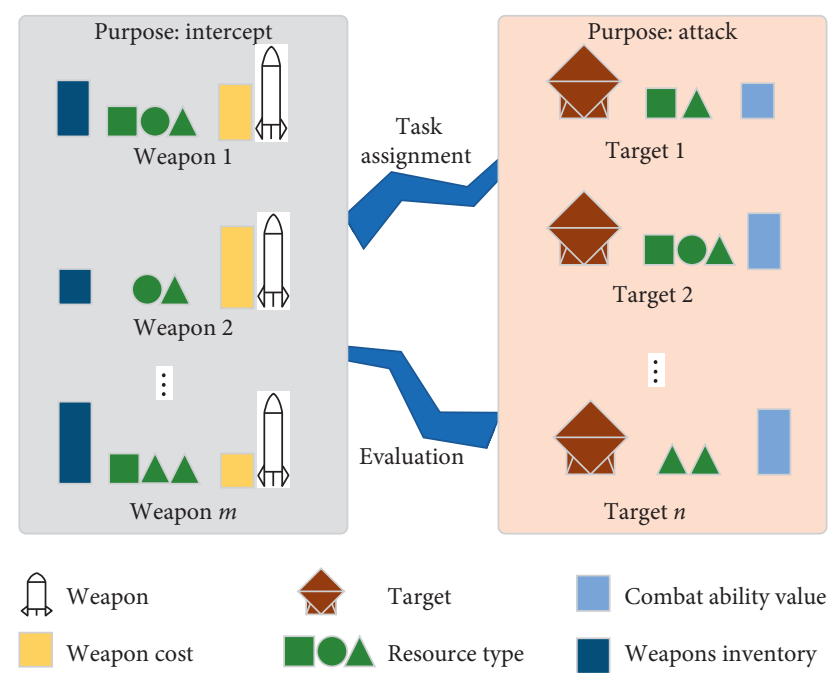

Figure 1: Drawing of DWTA.

assign $m$ weapons to $n$ targets in every stage, and then the evaluation of the combat result is implemented, which allows us to decide whether to go on the next weapon target assignment. Actually, this DWTA model belongs to shoot-look-shoot variant.

Assumption 4. The evaluation process is deterministic as long as the solution of WTA is acquired, which allows us to decide the next procedure according to the cessation conditions. The detail of cessation conditions will be described later.

2.2. Design of DWTA Formulation. After the description of the combat scenario in Figure 1, the detailed DWTA formulation will be introduced in this subsection, cost function constituted by consumed weapons and benefit function described by the combat ability value in each combat stage are defined at first, and the DWTA formulation is built based on a tradeoff between cost function and benefit function. Some notations used in this paper are described in Table 1.

In order to describe the cost of consumed weapons clearly, $g\left(x_{i}(t), j\right)$ is the auxiliary function of decision variable we defined, which depicts whether to assignweapon $i$ to target $j$ at stage $t$, the specific definition is as follows:

$$
\begin{gathered}
g\left(x_{i}(t), j\right)= \begin{cases}1, & \text { if } x_{i}(t)=j, \\
0, & \text { otherwise, }\end{cases} \\
\forall i \in W, j \in J, x_{i} \in J .
\end{gathered}
$$

Furthermore, we define the auxiliary function of combat feasibility $h(i, j, t)$ which is utilized to decide whether the weapon $i$ could intercept the target $j$ :

$$
\begin{gathered}
h(i, j, t)= \begin{cases}1, & \forall k=1, \ldots, K, \text { s.t. } r_{i k}(t) \geq q_{j k}(t), \\
0, & \text { otherwise, }\end{cases} \\
\forall i \in W, j \in J, t=1,2, \ldots, T,
\end{gathered}
$$

where $r_{i k}(t)$ is the number of resource $k$ contained by weapon $i$ at stage $t, q_{j k}(t)$ is the number of resource $k$ required by target $j$ at stage $t$, and both $r_{i k}(t)$ and $q_{j k}(t)$ are nonnegative integers. Weapon $i$ can be used to intercept target $j$ if and only if the available resource number of the weapon $i$ meets the requirement condition of target $j$.

Consequently, we get the cost function at stage $t$.

$$
S_{C}(X(t))=\sum_{j \in J} \sum_{i \in W} c_{i} g\left(x_{i}(t), j\right) h(i, j, t) .
$$

After the $t$ stage, the benefit function is equal to the sum of the combat ability value of all targets.

$$
S_{B}(X(t))=\sum_{j \in J} u_{j}(t)\left(\prod_{i \in W}\left(1-\omega_{i j}(t)\right)^{g\left(x_{i}(t), j\right) h(i, j, t)}\right) .
$$

Hence, we have built the cost function and the benefit function, weight strategy is adopted to balance the two conflicting objectives, and the DWTA objective function at stage $t$ is acquired as follows:

$$
\begin{aligned}
S(X(t))= & \alpha_{1} S_{C}(X(t))+\alpha_{2} S_{B}(X(t)) \\
= & \alpha_{1} \sum_{j \in J} \sum_{i \in W} c_{i} g\left(x_{i}(t), j\right) h(i, j, t)+\alpha_{2} \sum_{j \in J} u_{j}(t) \\
& \cdot\left(\prod_{i \in W}\left(1-\omega_{i j}(t)\right)^{g\left(x_{i}(t), j\right) h(i, j, t)}\right),
\end{aligned}
$$

where $\alpha_{1}+\alpha_{2}=1, \alpha_{1}$ and $\alpha_{2}$ are the weight parameters. Let $\alpha_{1}=0$, which means destroying targets regardless of cost, and let $\alpha_{2}=0$, which means destroying targets at the lowest cost.

The constraint conditions of the WTA problem are depicted in the following formulas:

$$
\begin{aligned}
& \sum_{j \in J} g\left(x_{i}(t), j\right)=1, \quad \forall i \in W, t=1, \ldots, T, \\
& \sum_{j \in J} g\left(x_{i}(t), j\right) \leq v_{i}(t), \quad \forall i \in W, t=1, \ldots, T, \\
& x_{i}(t) \in J \text { and } x_{i}(t) \text { is a positive integer. }
\end{aligned}
$$

Constraint (6) depicts that each weapon must be assigned to one of the targets at stage $t$. Constraint (7) depicts that it is necessary to stockpile enough weapons to complete weapon target assignment. Constraint (8) limits the range of $x_{i}(t)$; that is, $x_{i}(t)$ is a positive integer and belongs to the set $J$. There are two noticeable situations as follows:

Situation 1. A certain type of weapon is exhausted.

Situation 2. A certain type of weapon does not satisfy the resource requirement condition of any target.

In these two conditions which mean that this weapon cannot intercept any target, in order to deal with the two situations, we set up a new hypothetical target named target $n+1$ and get a new target set $\widetilde{J}=J+\{n+1\}$, we can assign this type of weapon to target $n+1$, and we set 
TABLE 1: Notation description.

$n$ : number of targets, $J$ is the target set and $J=\{1, \ldots, n\}$, target $j(j \in J)$ represents the $j$-th target

$n+1$ : hypothetical target, $\widetilde{J}$ is the target set and $\widetilde{J}=J+\{n+1\}$

$m$ : number of weapon types, $W$ is the weapon set and $W=\{1,2, \ldots, m\}$, weapon $i(i \in W)$ represents the $i-$ th type of weapon

$T$ : final combat stage, $t$ represents the $t$ stage and $t=1, \ldots, T$

$K$ : number of the resource types, $k$ represents the $k$-th kind of resource and $k=1, \ldots, K$

$u_{j}(t)$ : combat ability value of target $j$ at stage $t$

$c_{i}$ : unit cost of weapon $i$

$v_{i}(t)$ : inventory quantity of weapon $i$ at stage $t$

$\mathbf{X}(t)=\left[x_{i}(t)\right]_{m \times 1}$ : decision vector at stage $t, x_{i}(t)$ is the $i$-th decision variable $g\left(x_{i}(t), j\right)$ : auxiliary function of decision variable at stage $t$

$h(i, j, t)$ : auxiliary function of combat feasibility at stage $t$

$\mathbf{Q}(t)=\left[q_{j k}(t)\right]_{n \times K}:$ resources matrix of targets at stage $t$

$\mathbf{R}(t)=\left[r_{i k}(t)\right]_{m \times K}$ : resource matrix of weapons at stage $t$

$\boldsymbol{\Omega}(t)=\left[\omega_{i j}(t)\right]_{m \times n}:$ kill probability matrix at stage $t$

$S_{C}(\mathbf{X}(t))$ : cost of consumed weapons at stage $t$

$S_{B}(\mathbf{X}(t))$ : combat ability values of targets at stage $t$

$S(\mathbf{X}(t))$ : objective function at stage $t$

$\alpha_{1}, \alpha_{2}$ : weight parameters

$\varsigma^{*}$ : expected combat purpose

$\mathrm{X}^{*}$ : optimum solution and $S^{*}$ is the corresponding objective function value

$T_{\mathrm{CE}}$ : iteration number of CE method, and $T_{\mathrm{CE}}(t)$ is the iteration number of CE method at stage $t$

$D$ : iteration number of GA and PSO method

Num: population size of GA and PSO method

NMC: sample size of MC method

$g\left(x_{i}(t), j\right)=0, \forall x_{i}(t)=n+1, i \in W$. It is an excellent technique to deal with these two situations. Consequently, we can summarize that the DWTA formulation at stage $t$

$$
\left\{\begin{array}{l}
\min _{X(t)} S(X(t))=\alpha_{1} S_{C}(X(t))+\alpha_{2} S_{B}(X(t)) \\
=\alpha_{1} \sum_{j \in \widetilde{J}} \sum_{i \in W} c_{i} g\left(x_{i}(t), j\right) h(i, j, t)+\alpha_{2} \sum_{j \in \widetilde{J}} u_{j}(t) \\
\cdot\left(\prod_{i \in W}\left(1-\omega_{i j}(t)\right)^{g\left(x_{i}(t), j\right) h(i, j, t)}\right), \\
\sum_{j \in \widetilde{J}} g\left(x_{i}(t), j\right)=1, \quad \forall i \in W, \\
\sum_{j \in \widetilde{J}} g\left(x_{i}(t), j\right) \leq v_{i}(t), \quad \forall i \in W, \\
x_{i}(t) \in \widetilde{J} \text { and } x_{i}(t) \text { is a positive integer. }
\end{array}\right.
$$

\section{Algorithm Design Based on Cross-Entropy}

3.1. The Cross-Entropy Method. Originally, CE method is based on importance sampling and used to estimate the probability of rare event, and Rubinstein [22] put forward an optimization method based on the idea of CE, which could solve the discrete multiextreme value problems. Consider the following general minimization problem: let $S$ be a realvalued function on $\chi$, where $\chi$ is a finite set of states. Our goal is to find the minimum of $S$ over $\chi$, which is denoted as $\gamma^{*}$, and $X^{*}$ is the corresponding state.

$$
S\left(X^{*}\right)=\gamma^{*}=\min _{X \in \chi} S(X) .
$$

The basic idea of CE method applied to formula (10) is to associate an estimation problem with the optimization problem. In that case, the optimization problem is converted into an estimation problem with probability density function (pdf) $f(\cdot, \beta)$.

$$
l=\mathrm{P}_{\beta}(S(X) \leq \gamma)=\mathrm{E}_{\beta} I_{\{S(X) \leq \gamma\}}
$$

where $\gamma$ is a value close to $\gamma^{*}, \mathrm{P}_{\beta}$ is the probability measure, $\mathrm{E}_{\beta}$ denotes the corresponding expectation operator, and $I_{\{S(X) \leq \gamma\}}$ is the indicator function.

$$
I_{\{S(X) \leq \gamma\}}= \begin{cases}1, & \text { if } S(X) \leq \gamma \\ 0, & \text { if } S(X)>\gamma .\end{cases}
$$

A better way to estimate $l$ is to use another pdf $g$, and we can represent $l$ as in the following formula:

$$
l=\int I_{\{S(X) \leq \gamma\}} \frac{f(X)}{g(X)} g(X) \mathrm{d} x=\mathrm{E}_{\beta} I_{\{S(X) \leq \gamma\}} \frac{f(X)}{g(X)} .
$$

An unbiased estimator of $l$ is depicted in the following formula:

$$
\widehat{l}=\frac{1}{N} \sum_{i=1}^{N} I_{\left\{S\left(X_{i}\right) \leq \gamma\right\}} \frac{f\left(X_{i} ; \beta\right)}{g\left(X_{i}\right)},
$$

where $X_{1}, X_{2}, \ldots, X_{N}$ is generated from pdf $g$.

It is well known that the best way to estimate $l$ is to use the change of measure with the pdf $g^{*}(X)$ :

$$
g^{*}(X)=\frac{I_{\left\{S\left(X_{i}\right) \leq \gamma\right\}} f(X ; \beta)}{l} .
$$

In order to choose the pdf $g^{*}$ from the family of pdf $\{f(\cdot ; \delta)\}$, the idea is to choose the reference parameter $\delta$ such that the distance between the densities gand $f(\cdot ; \delta)$ is minimal. Minimizing the Kullback-Leibler distance between the densities $g$ and $f(\cdot ; \delta)$ is equivalent to the following formula: 


$$
\begin{aligned}
\min _{\delta} D(\delta)= & \min _{\delta} \mathrm{E}_{g^{*}} \ln \frac{g^{*}(X)}{f(X ; \delta)}=\min _{\delta} \int g^{*}(X) \ln g^{*}(X) \mathrm{d} X \\
& -\int g^{*}(X) \ln f(X ; \delta) \mathrm{d} X .
\end{aligned}
$$

It is obvious that minimizing problem (16) is equivalent to solving the minimization problem.

$$
\begin{gathered}
\min _{\delta}-\int g^{*}(X) \ln f(X ; \delta) \mathrm{d} X=\min _{\delta}-\int \frac{I_{\left\{s\left(X_{i}\right) \leq \gamma\right\}} f(X ; \beta)}{l} \\
\cdot \ln f(X ; \delta) \mathrm{d} X .
\end{gathered}
$$

Finally, formula (17) is converted into the following formula:

$$
\min _{\delta}-\mathrm{E}_{\beta} I_{\left\{S\left(X_{i}\right) \leq \gamma\right\}} \ln f(X ; \delta)=\min _{\delta}-\frac{1}{N} \sum_{i=1}^{N} I_{\left\{S\left(X_{i}\right) \leq \gamma\right\}} \ln f\left(X_{i} ; \delta\right),
$$

where $X_{1}, X_{2}, \ldots, X_{N}$ is the random sample from $f(\cdot ; \beta)$. In typical applications, the function $D(\delta)$ in formula (16) is convex and differentiable with respect to $\delta$ [27]. Thus, we can get the solution of (18) from the following equation:

$$
-\frac{1}{N} \sum_{i=1}^{N} I_{\left\{S\left(X_{i}\right) \leq \gamma\right\}} \nabla \ln f\left(X_{i} ; \delta\right)=0
$$

where the gradient is with respect to $\delta$.

The idea of CE method is to construct simultaneously two sequences of levels $\gamma_{1}, \gamma_{2}, \ldots, \gamma_{T_{C F}}$ and parameters $\delta_{1}, \delta_{2}, \ldots, \delta_{T_{\mathrm{CE}}}$, where $\gamma_{T_{\mathrm{CE}}}$ is close to the optimal $\gamma^{*}$ and $\delta_{T_{\mathrm{CE}}}$ is the corresponding parameter of pdf $g^{*}$. In other words, each iteration of the CE method has two main phases. In the first phase, $\gamma_{t_{\mathrm{CE}}}$ is updated, and in the second $\delta_{t_{\mathrm{CE}}}$ is updated. To be specific, starting $\delta_{0}=\beta$, we can obtain the two sequences as follows:

Phase 1: adaptive updating of $\gamma_{t_{\mathrm{CE}}}$

For a fixed $\delta_{t_{\mathrm{CE}}-1}$, let $\gamma_{t_{\mathrm{CE}}}$ be a $\theta$-quantile of $S(X)$ under $\delta_{t_{\mathrm{CE}}-1}$; that is, $\gamma_{t_{\mathrm{CE}}}$ satisfies

$$
\mathrm{P}_{\beta}(S(X) \leq \gamma) \geq \theta,
$$

where $X$ is generated from $f\left(\cdot ; \delta_{t_{\mathrm{CE}}-1}\right)$.

A simple estimator $\widehat{\gamma}_{t_{\mathrm{CE}}}$ of $\gamma_{t_{\mathrm{CE}}}$ can be obtained by drawing a random sample $X_{1}, X_{2}, \ldots, X_{N}$ from $f\left(\cdot ; \delta_{t_{\mathrm{CE}}-1}\right)$, calculating the performances $S\left(X_{i}\right)$ for all $I$, ordering them from the smallest to the biggest, $S_{(1)} \leq S_{(2)} \leq \cdots \leq S_{(N)}$, and evaluating the $\theta$-quantile as

$$
\widehat{\gamma}_{t_{\mathrm{CE}}}=S(\lceil\theta N\rceil) \text {. }
$$

Phase 2: adaptive updating of $\delta_{t_{\mathrm{CE}}}$

For fixed $\gamma_{t_{\mathrm{CE}}}$ and $\delta_{t_{\mathrm{CE}}-1}$, derive $\delta_{t_{\mathrm{CE}}}$ from the solution of (19), to be specific
3.2. Solving DWTA Problem Based on CE Method. In this paper, our purpose is to apply CE method to solve the DWTA problem modeled above, and interested readers can get detailed theory of CE method in $[22,28]$. In order to solve formulation (9) at stage $t$, we construct the following discrete Probability Distribution Matrix (PDM) $\mathbf{M}(t)$ :

$$
\mathbf{M}(t)=\left(\begin{array}{cccc}
p(1 \mid 1, t) & \cdots & p(n \mid 1, t) & p(n+1 \mid 1, t) \\
p(1 \mid 2, t) & \cdots & p(n \mid 2, t) & p(n+1 \mid 2, t) \\
\vdots & & \vdots & \vdots \\
p(1 \mid m, t) & \cdots & p(n \mid m, t) & p(n+1 \mid m, t)
\end{array}\right)_{m \times(n+1)}
$$

where the element $p(j \mid i, t)$ in PDM denotes the probability of allocating weapon $i$ to target $j$ at stage $t$, and if every element $p(j \mid i, t)$ belongs to $\{0,1\}$, we can get the final solution according to the PDM. Furthermore, the PDM $\mathbf{M}(t)$ satisfies the following constraint condition:

$$
\sum_{j \in \widetilde{J}} p(j \mid i, t)=1, \quad \forall i \in W, t=1, \ldots, T,
$$

where the set $\widetilde{J}=J+\{n+1\}$ because we use target $n+1$ to deal with Situation 1 and Situation 2, and it is obvious that constraint condition (6) is equivalent to constraint condition (23). It is worth noting that initializing the $\operatorname{PDM~} \mathbf{M}_{0}(t)$ at stage $t$ according to the following formula can assure that sample $\mathbf{X}$ generated from $\operatorname{PDM} \mathbf{M}(t)$ meets the resources requirement:

$$
p(j \mid i, t)= \begin{cases}\frac{1}{\sum_{j \in J} h(i, j, t)}, & \text { if } h(i, j, t) \neq 0, \\ 0, & \text { if } h(i, j, t)=0,\end{cases}
$$

$$
\forall i \in W, t=1, \ldots, T \text {. }
$$

According to the PDM $\mathbf{M}(t)$ at stage $t$, we can get the probability distribution function $f(\mathbf{X}(t) ; \mathbf{M}(t))$ :

$$
f(\mathbf{X}(t) ; \mathbf{M}(t))=\prod_{i \in W} p\left(x_{i}(t) \mid i, t\right),
$$

where $p\left(x_{i}(t) \mid i\right)$ is the element of column $x_{i}(t)$ in line $i$ of $\operatorname{PDM} \mathbf{M}(t)$.

Furthermore, we can get the following probability distribution function:

$$
f(\mathbf{X}(t) ; \mathbf{M}(t))=\prod_{i \in W} p\left(x_{i}(t) \mid i, t\right)=\prod_{i \in W} \prod_{j \in J} p(j \mid i, t)^{g\left(x_{i}(t), j\right)},
$$

where formulas (25) and (26) are equivalent, and the reader can refer to Appendix for details.

In each iteration process of CE method, $\mathbf{X}^{1}(t), \ldots, \mathbf{X}^{N}(t)$ are $N$ samples generated from the $\operatorname{PDM} \mathbf{M}(t)$, and we can compute the objective function values $S\left(\mathbf{X}^{l}(t)\right), \forall l=1, \ldots, N$, and then we sort the $N$ 
objective function values in ascending order, and we can get the sequence of objective function values $S\left(\mathbf{X}^{(1)}(t)\right) \leq \cdots \leq S\left(\mathbf{X}^{(N)}(t)\right)$ and the sequence of samples $\mathbf{X}^{(1)}(t), \ldots, \mathbf{X}^{(N)}(t)$; let $H=\lfloor\theta N\rfloor$, and \lfloor\rfloor is the symbol of rounding down, which is the $\theta$-quantile. We select $H$ samples corresponding to the $H$ minimum objective function values; that is, $\mathbf{X}^{(1)}(t), \ldots, \mathbf{X}^{(H)}(t)$, to update the $\operatorname{PDM} \mathbf{M}(t)$. We can get the following optimization problem according to formula (18):

$$
\left\{\begin{array}{l}
\min _{\mathbf{M}(t)}-\sum_{s=1}^{H} \ln (f(\mathbf{X}(t) ; \mathbf{M}(t))), \\
\text { s.t. } \sum_{j \in \widetilde{J}} p(j \mid i, t)=1, \quad \forall i \in W, t=1, \ldots, T, \\
p(j \mid i, t) \geq 0, \quad \forall i \in W, j \in \widetilde{J}, t=1, \ldots, T .
\end{array}\right.
$$

According to formula (26), we can simplify formula (27):

$$
\begin{aligned}
\min _{\mathbf{M}(t)}-\sum_{s=1}^{H} \ln \left(f\left(\mathbf{X}^{s}(t) ; \mathbf{M}(t)\right)\right) & =\min _{\mathbf{M}(t)}-\sum_{s=1}^{H} \ln \left(\prod_{i \in W} p\left(x_{i}^{s}(t) \mid i, t\right)\right) \\
& =\min _{\mathbf{M}}-\sum_{s=1}^{H} \ln \left(\prod_{i \in W} \prod_{j \in \widetilde{J}} p(j \mid i, t)^{g\left(x_{i}^{s}(t), j\right)}\right) \\
& =\min _{\mathbf{M}}-\sum_{s=1}^{H} \sum_{i=1}^{m} \sum_{j=1}^{n+1} g\left(x_{i}^{s}(t), j\right) \ln (p(j \mid i, t)) .
\end{aligned}
$$

In order to simplify the description, let $r_{j i}(t)=p(j \mid i, t)$, $b_{j i}^{s}(t)=g\left(x_{i}^{s}(t), j\right)$, and then optimization problem (27) can be rewritten as follows:

$$
\left\{\begin{array}{l}
\min _{M(t)}-\sum_{s=1}^{H} \sum_{i=1}^{m} \sum_{j=1}^{n+1} b_{j i}^{s}(t) \ln \left(r_{j i}(t)\right), \\
\text { s.t. } \sum_{j=1}^{n+1} r_{j i}(t)=1, \quad \forall i \in W, t=1, \ldots, T, \\
r_{j i}(t) \geq 0, \quad \forall i \in W, j \in \widetilde{J}, t=1, \ldots, T .
\end{array}\right.
$$

It is obvious that problem (29) belongs to a convex optimization problem, and we can construct the Lagrange function as follows:

$$
\begin{aligned}
L\left(r_{j i}(t), \lambda_{i}(t), \alpha_{j i}(t)\right)= & -\sum_{s=1}^{H} \sum_{i=1}^{m} \sum_{j=1}^{n+1} b_{j i}^{s}(t) \ln \left(r_{j i}(t)\right) \\
& +\sum_{i=1}^{m} \lambda_{i}(t)\left(\sum_{j=1}^{n+1} r_{j i}(t)-1\right) \\
& +\sum_{i=1}^{m} \sum_{j=1}^{n+1} \alpha_{j i}(t)\left(-r_{j i}(t)\right) .
\end{aligned}
$$

According to Karush-Kuhn-Tucker conditions, we can get the following formula:

$$
\left\{\begin{array}{l}
-\frac{\sum_{s=1}^{H} b_{j i}^{s}(t)}{r_{j i}(t)}+\lambda_{i}(t)-\alpha_{j i}(t)=0 \\
\alpha_{j i}(t)\left(-r_{j i}(t)\right)=0 \\
\sum_{j=1}^{n+1} r_{j i}(t)-1=0 \\
\alpha_{j i}(t) \geq 0 \\
-r_{j i}(t) \leq 0 \\
\lambda_{i}(t)>0
\end{array}\right.
$$

and the following formula is derived according to the upper formula (31):

$$
\left\{\begin{array}{l}
r_{j i}(t)=\frac{\sum_{s=1}^{H} b_{j i}^{s}(t)}{H} \\
\alpha_{j i}(t)=0 \\
\lambda_{i}(t)=H
\end{array}\right.
$$

Then, the element $p(j \mid i, t)$ in PDM $\mathbf{M}(t)$ can be updated according to the following formula: 


$$
p(j \mid i, t)=\frac{\sum_{s=1}^{H} b_{j i}^{s}(t)}{H}=\frac{\sum_{s=1}^{H} g\left(x_{i}^{s}(t), j\right)}{H} .
$$

Before the cessation conditions of the DWTA are analyzed, we give the updated formula of the remaining weapon inventory and the remaining combat ability value after the $t$ stage.

$$
\begin{aligned}
& v_{i}(t+1)=v_{i}(t)-\sum_{j \in J} g\left(x_{i}(t), j\right), \\
& u_{j}(t+1)=u_{j}(t)\left(\prod_{i \in W}\left(1-\omega_{i j}(t)\right)^{g\left(x_{i}(t), j\right) h(i, j, t)}\right),
\end{aligned}
$$

where $j \in \widetilde{J}, i \in W, t=1, \ldots, T$.

The cessation conditions of the DWTA include two points:

Cessation condition 1: all available weapons are exhausted

Cessation condition 2: the combat purpose is implemented, which means the percentage of the remaining combat ability value is less than the preselected percentage $\varsigma^{*}$

Consequently, we can get the DWTA algorithm based on CE method as follows (Algorithm 1).

3.3. Complexity Analysis. Let $N$ denote the sample size, $T_{\mathrm{CE}}$ denote the iteration number of $\mathrm{CE}$ method, $m$ denote the number of weapon types, and $T$ denote the final combat stage. The computational cost of $\mathrm{CE}$ method includes four parts: the initialization $\left(C_{\text {ini }}\right)$, sample $\left(C_{\text {sample }}\right)$, sort $\left(C_{\text {sort }}\right)$, and update $\left(C_{\text {update }}\right)$; the computational complexity of $\mathrm{CE}$ method can be denoted as $C_{\mathrm{CE}}=C_{\text {ini }}+C_{\text {sample }}+C_{\text {sort }}+$ $C_{\text {update }}$, to be specific, $C_{\mathrm{CE}}=m+T_{\mathrm{CE}}[m N+N+m \theta N]$; therefore, the time complexity of the $\mathrm{CE}$ method can be computed as $O\left(T_{\mathrm{CE}} m N\right)$, and the time complexity of the DWTA algorithm based on CE method can be denoted as $O\left(T T_{\mathrm{CE}} m N\right)$. Let Num denote the population size of PSO and GA. Let $D$ denote the iteration number of PSO and GA, and we can get the complexity of DWTA algorithm based on PSO ang GA denoted as $O(\mathrm{TD} \cdot \mathrm{Num})$.

\section{DWTA Simulations}

4.1. Parameter Setting for DWTA. The following contents in this subsection include three parts: parameters setting rules of combat scenario, parameters selection of algorithms, performance index design and parameters setting of combat scenario. The first part is the parameters setting rules of combat scenario which is depicted in Table 2. Both the number of weapon types $(m)$ and the number of targets $(n)$ are integers between 1 and 80 , that is, $m, n \in[1,80]$. The combat ability value of every target at the first stage is a random number between 70 and 200, that is, $u_{j}(1) \in(70,200), \forall j \in J$; it is worth noting that the combat ability value of the hypothetical target is equal to zero, that is, $u_{n+1}(t)=0, \forall t=1, \ldots, T$. The cost of every single weapon is a random number between 20 and 80 , that is, $c_{i} \in(20,80), \forall i \in W$. The kill probability of weapon $i$ to target $j$ is a random number between 0.5 and 1 , that is, $\omega_{i j}(t) \in(0.5,1) \cdot q_{j k}(t)$ is integer between 0 and 3. $r_{i k}(t)$ is integer between 0 and 5 . The expected combat purpose $\varsigma^{*}$ is a random number between 0 and 1 .

Subsequently, we give the scheme of parameter selection of CE, PSO, GA, and MC methods in Table 3. The CE method includes two parameters, sample size and quantile, and the main parameters of PSO and GA methods are population size Num and iteration number $D$, and we set sample size of MC method equal to 5e4 in the simulation.

The third part is performance index design depicted in Table 4 which measures the solution quality of different algorithms. The percentage between the sum of the cost of weapon consumed in all stages and the total value of weapons, denoted as PSCWC, is to depict combat costs in whole combat.

$$
\operatorname{PSCWC}=\frac{\sum_{t=1}^{T} \sum_{j \in \widetilde{J}} \sum_{i \in W} c_{i} g\left(x_{i}^{*}(t), j\right)}{\sum_{i \in W} c_{i} v_{i}(1)} \times 100 \% .
$$

The index PRCAVT denotes the percentage between the remaining combat ability value of targets at $T$ stage and the initial combat value of targets, which measures whether to achieve the expected combat purpose.

$$
\text { PRCAVT }=\frac{\sum_{j \in J} u_{j}(T)}{\sum_{j \in J} u_{j}(1)} \times 100 \% .
$$

The index $\mathrm{CI}$ is the product of the index PRCAVT and the index PSCWC. The index avg-PSCWC is the average of PSCWC corresponding to the six DWTA problems. The index avg-PRCAVT is the average of PRCAVT corresponding to the six DWTA problems. The computation time (CT) is used to depict the solving speed of different algorithms.

Finally, we categorize the number of weapon stockpiles and weight parameters of DWTA model into the following four situations so as to cover different combat scenarios.

Scenario 1. Weapon stockpiles are sufficient, and the weapon costs are important the same as with the combat benefits.

$$
\begin{aligned}
v_{i} & =\lfloor 6+4 \cdot \operatorname{rand}(m, 1)\rfloor, \\
\alpha_{1} & =\alpha_{2}=0.5 .
\end{aligned}
$$

Scenario 2. Weapon stockpiles are sufficient, and the combat benefits are more important than the weapon costs.

$$
\begin{aligned}
v_{i} & =\lfloor 6+4 \cdot \operatorname{rand}(m, 1)\rfloor, \\
\alpha_{1} & =0.1, \\
\alpha_{2} & =0.9 .
\end{aligned}
$$

Scenario 3. Weapon stockpiles are not sufficient, and the weapon costs are important the same as with the combat benefits. 
Input: $t, n, m, K, u_{j}, c_{i}, v_{i}, \zeta^{*}, \mathbf{Q}, \mathbf{R}, \boldsymbol{\Omega}, \theta, N$

Output: $T, S^{*}, \mathbf{X}^{*}$

(1) Let $t=1$, begin the dynamic weapon target assignment.

(2) Compute the initial PDM $\mathbf{M}_{0}(t)$ according to formula (24) and let $t_{\mathrm{CE}}=0$.

(3) Generate $N$ samples $\theta=0.1$ according to PDM $\mathbf{M}_{t_{\mathrm{CE}}}(t)$.

(4) Compute the objective function values $S\left(\mathbf{X}^{1}(t)\right), \ldots, S\left(\mathbf{X}^{N}(t)\right)$.

(5) Sort the $N$ objective function values in ascending order and select $H$ samples corresponding to the $H$ minimum objective function values.

(6) Update the PDM $\mathbf{M}_{t_{\mathrm{CE}}}(t)$ using the $H$ samples according to formula (33).

(7) If all elements of PDM $\mathbf{M}_{t_{\mathrm{CE}}}(t)$ belong to $\{0,1\}$, set $T_{\mathrm{CE}}=t_{\mathrm{CE}}$, output the optimum $\mathbf{X}^{*}$ and $\mathbf{S}^{*}$, else let $t_{\mathrm{CE}}=t_{\mathrm{CE}}+1$, goto step 3 until the $\mathrm{CE}$ method achieves the convergence conditions.

(8) Update the remaining combat ability value of every target $u_{j}(t+1)$ and the remaining weapon inventory $v_{i}(t+1)$.

(9) If any of the two cessation conditions is reached, let $T=t$, output $T, S^{*}(t), \mathbf{X}^{*}(t)$. Otherwise, let $t=t+1$, go to step 2.

Algorithm 1: The DWTA algorithm based on CE method.

TABLE 2: Combat scenario parameter setting rules.

\begin{tabular}{lr}
\hline Parameter & Parameter setting rules \\
\hline$n$ & The number of weapon types $(m)$ is integer between 1 and 80 \\
$m$ & The number of targets $(n)$ is integer between 1 and 80 \\
$K$ & The number of the resource types is integer between 1 and 9 \\
$u_{j}(1)$ & The combat ability value of target $j$ at the first stage is a random number between 70 and 200 and $u_{n+1}(1)=0$ \\
$c_{i}$ & The cost of every single weapon is a random number between 20 and 80 \\
$v_{i}(1)$ & The inventory quantity of weapon $i$ at the first stage is integer between 0 and 10 \\
$q_{j k}(t)$ & The quantity of resource $k$ required by target $j$ at stage $t$ is integer between 0 and 3 \\
$r_{i k}(t)$ & The quantity of resource $k$ contained by weapon $i$ at stage $t$ is integer between 0 and 5 \\
$\omega_{i j}(t)$ & The kill probability of weapon $i$ to target $j$ is a random number between 0.5 and 1 \\
$\varsigma^{*}$ & The expected combat purpose is a random number between 0 and 1
\end{tabular}

TABle 3: Parameters setting of different methods.

\begin{tabular}{lcc}
\hline Method & Parameter & Parameter setting rules \\
CE & $N$ & The sample size of CE method is integer between 50 and 200 \\
PSO & $\theta$ & The quantile $\theta$ is a random number between 0 and 1 \\
& Num & The population size of PSO is integer between 50 and 200 \\
GA & Num & The iteration number of PSO is integer between 50 and 100 \\
MC & $D$ & The population size of GA is integer between 50 and 200 \\
& NMC & The iteration number of GA is integer between 50 and 100 \\
\end{tabular}

TABle 4: Description of several indexes.

Index
$\begin{aligned} & \text { PSCWC } \\ & \text { PRCAVT }\end{aligned}$ The percentage between the sum of the cost of weapon consumed in all stages and the total value of weapons
CI
$\begin{aligned} & \text { avg-PSCWC } \\ & \text { avg-PRCAVT }\end{aligned}$
The composite index which is the product of the index PRCAVT and the index PSCWC
The average of PSCWC corresponding to the six DWTA problems
The average of PRCAVT corresponding to the six DWTA problems
Computation time of all stages

TABLe 5: Six DWTA problems of different scales.

\begin{tabular}{lcccccc}
\hline No. & 1 & 2 & 3 & 4 & 5 & \\
\hline$n$ & 12 & 24 & 25 & 40 & 45 & 64 \\
$m$ & 16 & 16 & 32 & 32 & 54 & 54 \\
\hline
\end{tabular}




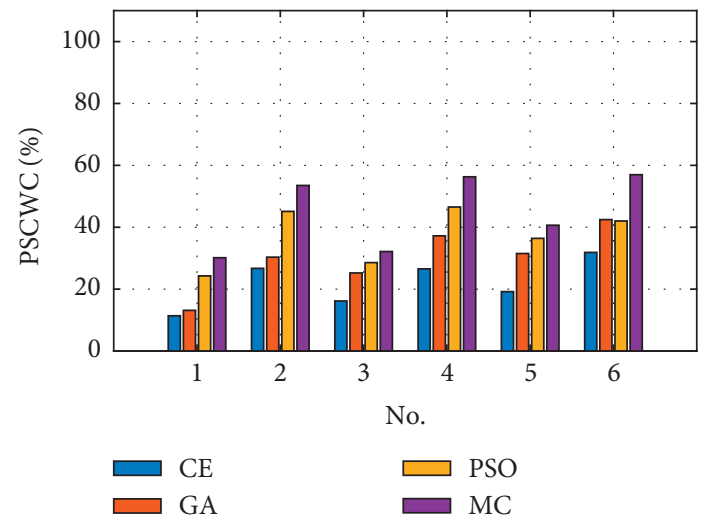

(a)

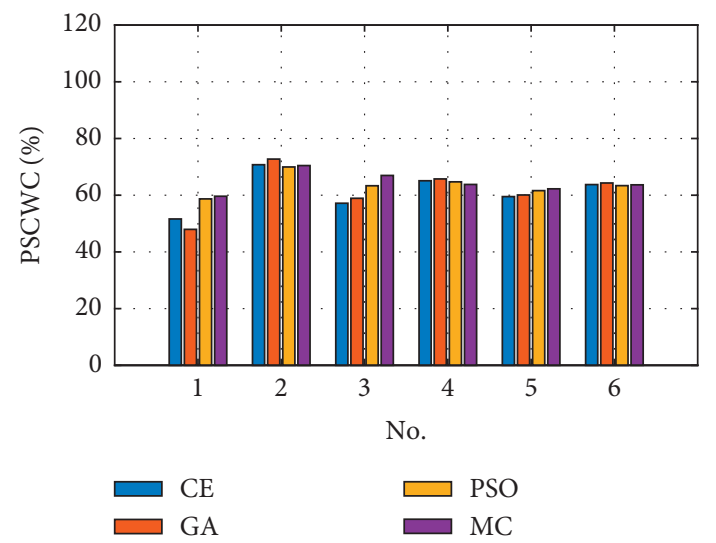

(c)

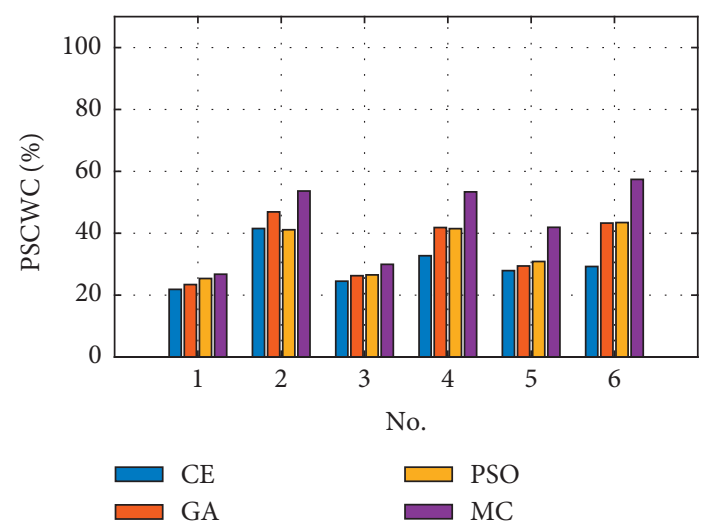

(b)

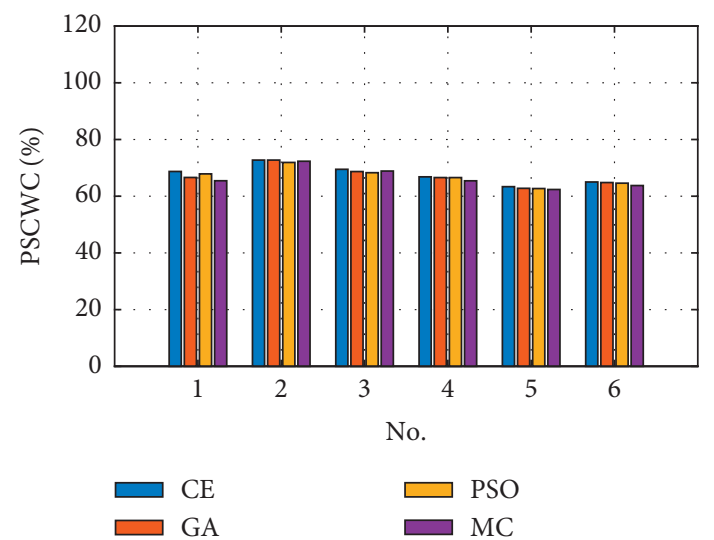

(d)

FIgURE 2: Comparative results of CE, GA, PSO, and MC methods with respect to PSCWC index. (a) Scenario 1. (b) Scenario 2. (c) Scenario 3. (d) Scenario 4.

$$
\begin{aligned}
v_{i} & =\lfloor 2+1 \cdot \operatorname{rand}(m, 1)\rfloor, \\
\alpha_{1} & =\alpha_{2}=0.5 .
\end{aligned}
$$

Scenario 4. Weapon stockpiles are not sufficient, and the combat benefits are more important than the weapon costs.

$$
\begin{aligned}
v_{i} & =\lfloor 2+1 \cdot \operatorname{rand}(m, 1)\rfloor, \\
\alpha_{1} & =0.1 \\
\alpha_{2} & =0.9 .
\end{aligned}
$$

It is worth noting that the same scale DWTA problems in Scenario 1 and Scenario 2 have the same parameters such as the inventory quantity of weapons and kill probability, and the same is true for Scenario 3 and Scenario 4. Besides, in order to describe the effectiveness of the DWTA model and the advantages of CE method in solving different scales of DWTA problems, we set up the following six scales DWTA problems. The choices of parameter $m$ and parameter $n$ are shown in Table 5.

We set the expected combat purpose $\varsigma^{*}=10 \%$ in advance, which depicts that we stop the combat task when the index PRCAVT is less than $\varsigma^{*}$; note that another stop condition is that all available weapons are exhausted. We set the relevant parameters of $\mathrm{CE}$, PSO, and GA methods as follows, $N=200$ and $\theta=0.1$, Num $=50, D=100$. In this paper, ten simulations of the same scale DWTA problem are carried out to calculate the statistical average of PSCWC, PRCAVT, and CT. All algorithms are implemented in an Intel Core I5 @ $3.06 \mathrm{GHz}$ processor with 8 GB RAM.

4.2. Results and Analysis. The results of simulation are presented in Figures 2-4 and Tables 6-8. What needs to be pointed out is that the smaller the indexes PSCWC and avgPSCWC are, the smaller the cost of weapons consumed is. The smaller the indexes PRCAVT and avg-PRCAVT are, the greater the combat benefits are. The smaller the index CI is, the better the solution quality is. The smaller the CT index is, the faster the solving speed is.

Figures 2(a), 3(a), and 4(a) depict the combat results in Scenario 1; the index PSCWC has the ascending sequence, that is, $\mathrm{CE}<\mathrm{GA}<\mathrm{PSO}<\mathrm{MC}$; furthermore, Figure $4(\mathrm{a})$ indicates that the value of index CI for CE method is the smallest among others, and from Tables 6 and 7, the indexes avg-PSCWC of CE, GA, PSO, and MC methods are $21.98 \%$, $29.98 \%, 37.16 \%$, and $44.96 \%$, and the indexes avg-PRCAVT of CE, GA, PSO, and MC methods are $7.97 \%, 8.28 \%, 7.18 \%$, and $6.85 \%$, which indicates that the solution of CE method is 


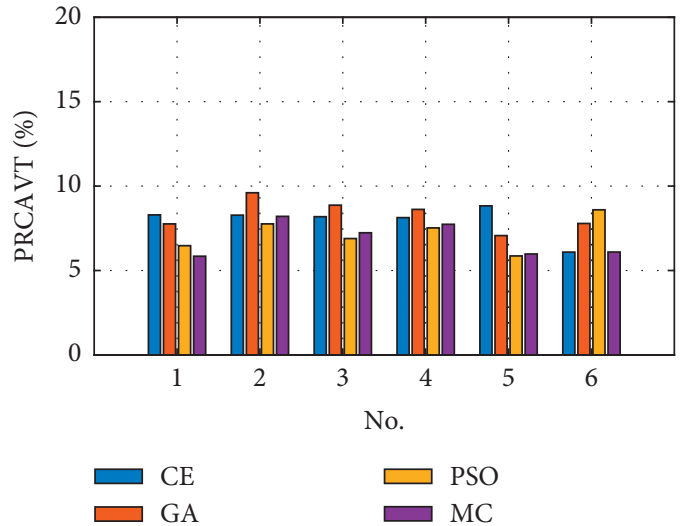

(a)

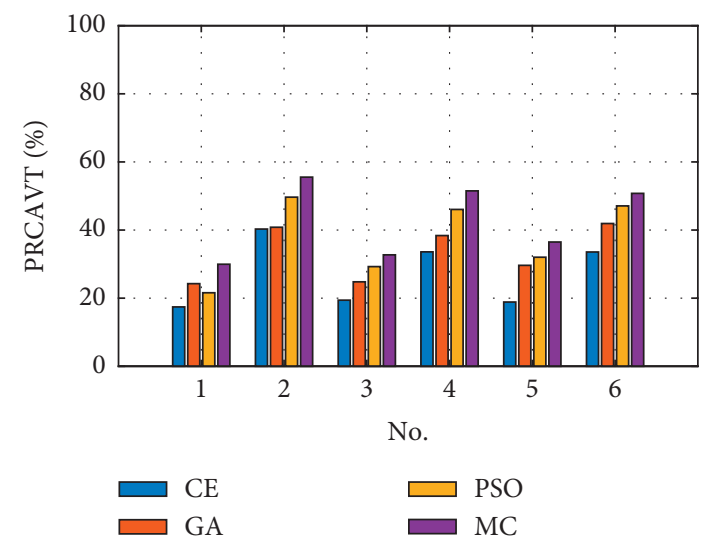

(c)

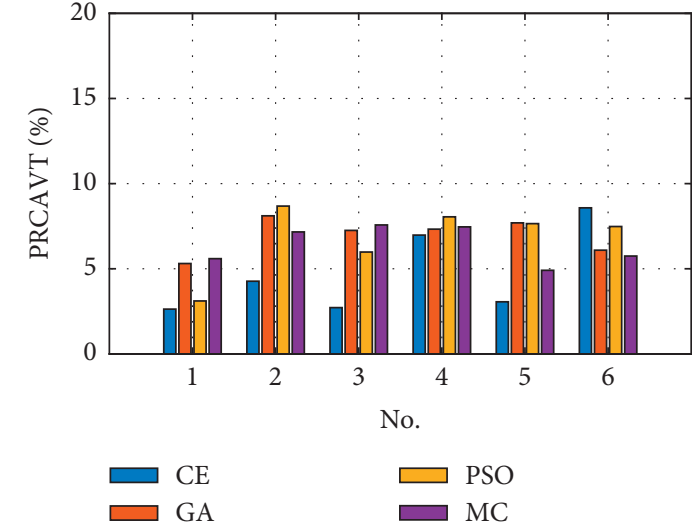

(b)

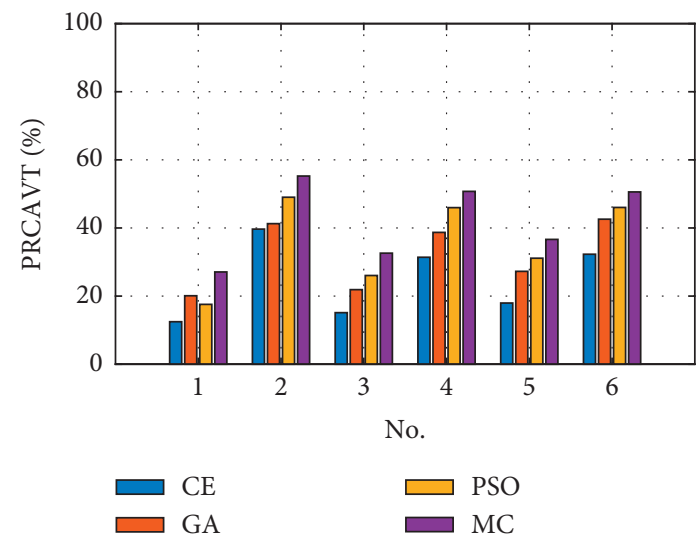

(d)

FIgure 3: Comparative results of CE, GA, PSO, and MC methods with respect to PSCWC index. (a) Scenario 1. (b) Scenario 2. (c) Scenario 3. (d) Scenario 4.

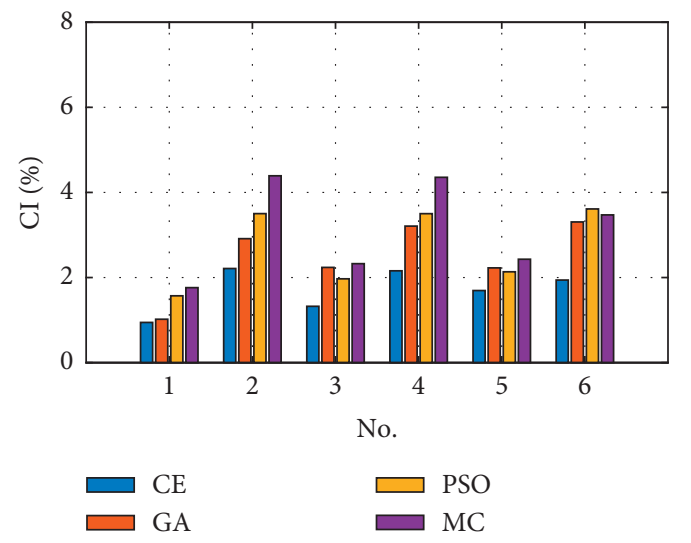

(a)

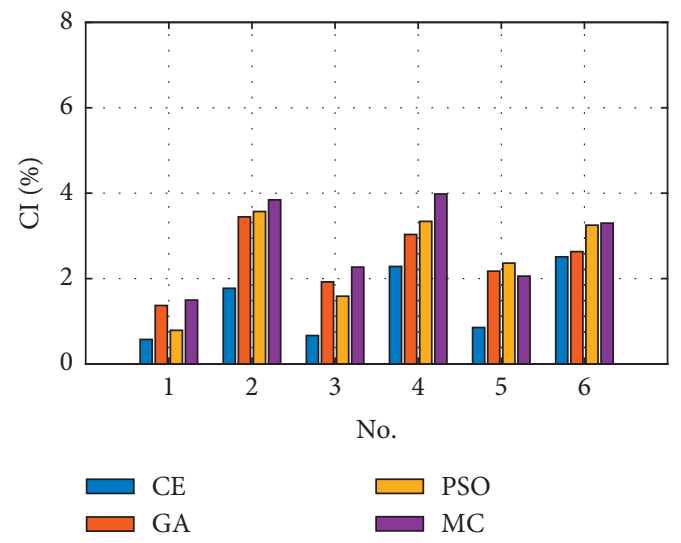

(b)

FIgURE 4: Continued. 


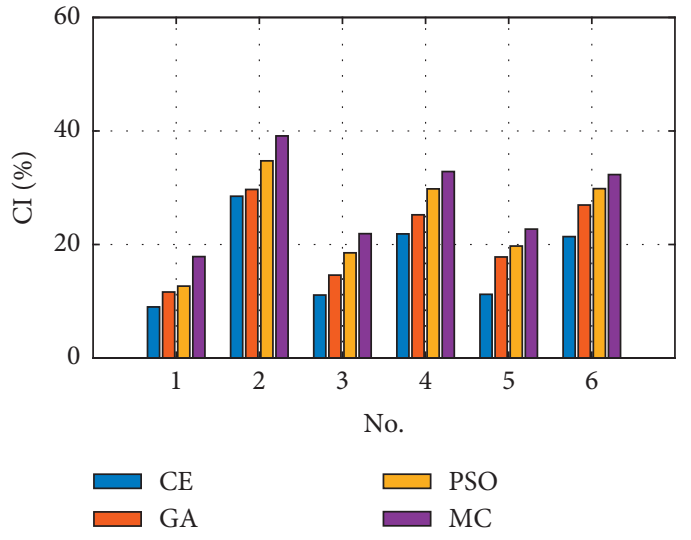

(c)

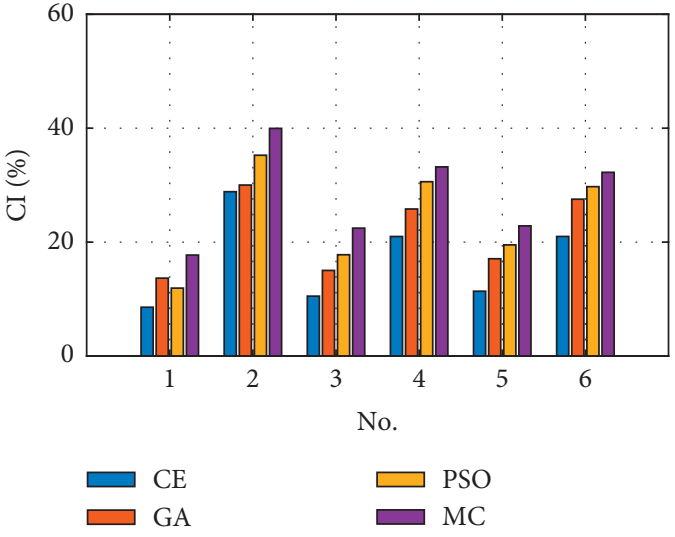

(d)

FIGURE 4: Comparative results of CE, GA, PSO, and MC methods with respect to the index CI. (a) Scenario 1. (b) Scenario 2. (c) Scenario 3. (d) Scenario 4.

TABLE 6: Comparative results of CE, GA, PSO, and MC methods with respect to the statistic average PSCWC index.

\begin{tabular}{lcccc}
\hline Method & CE (\%) & GA (\%) & PSO (\%) & MC (\%) \\
\hline Scenario 1 & 21.98 & 29.98 & 37.16 & 44.96 \\
Scenario 2 & 29.64 & 35.19 & 41.81 & 48.84 \\
Scenario 3 & 61.31 & 61.62 & 63.61 & 64.46 \\
Scenario 4 & 67.69 & 67.30 & 66.99 & 66.37
\end{tabular}

TABLe 7: Comparative results of CE, GA, PSO, and MC methods with respect to the index avg-PRCAVT.

\begin{tabular}{lcccc}
\hline Method & CE (\%) & GA (\%) & PSO (\%) & MC (\%) \\
\hline Scenario 1 & 7.97 & 8.28 & 7.18 & 6.85 \\
Scenario 2 & 4.71 & 6.96 & 6.83 & 6.41 \\
Scenario 3 & 27.19 & 33.30 & 37.61 & 42.82 \\
Scenario 4 & 24.82 & 31.97 & 35.96 & 42.15 \\
\hline
\end{tabular}

more excellent than these solutions of GA, PSO, and MC methods; that is, the expected combat purpose is achieved and multitudes of weapon costs are saved. The same conclusion can be reached in Scenario 2.

As for Scenario 3, the CE, GA, PSO, and MC methods have nearly the same PSCWC index depicted in Figure 2(c), and from Figure 3(c), the PRCAVT index has the ascending sequence, that is, $\mathrm{CE}<\mathrm{GA}<\mathrm{PSO}<\mathrm{MC}$, and Figure 4(c) indicates that the value of index CI for CE method is the smallest among others; furthermore, from Tables 6 and 7, the indexes avg-PSCWC of CE, GA, PSO, and MC methods are $61.31 \%$, $61.62 \%, 63.61 \%$, and $64.46 \%$, and the indexes avg-PRCAVT of CE, GA, PSO, and MC methods are $27.19 \%, 33.30 \%, 37.61 \%$, and $42.82 \%$. Consequently, compared with the GA, PSO, and MC methods, the assignment results of CE method are more effective in the condition that the number of weapons consumed is the same. According to the combat result of Scenario 3 , we can get the conclusion that the defense can get better combat benefits when using CE method to complete the DWTA task under the condition that the weapons are inadequate. The same conclusion can be reached in Scenario 4.
TABLE 8: Comparative results of CE, GA, PSO, and MC methods with respect to the index CT (s).

\begin{tabular}{lccccc}
\hline No. & Method & Scenario 1 & Scenario 2 & Scenario 3 & Scenario 4 \\
\hline \multirow{4}{*}{1} & CE & 0.51 & 0.26 & 0.13 & 0.14 \\
& GA & 6.78 & 2.59 & 1.23 & 1.22 \\
& PSO & 3.81 & 2.68 & 1.31 & 1.32 \\
& MC & 24.66 & 18.16 & 8.67 & 8.79 \\
\hline \multirow{4}{*}{2} & CE & 0.97 & 0.62 & 0.21 & 0.21 \\
& GA & 8.06 & 5.42 & 1.42 & 1.41 \\
& PSO & 7.65 & 5.49 & 1.79 & 1.8 \\
& MC & 50 & 46.97 & 11 & 11.03 \\
\hline \multirow{4}{*}{3} & CE & 1.43 & 1.02 & 0.53 & 0.54 \\
& GA & 10.8 & 5.94 & 2.88 & 2.93 \\
& PSO & 9.52 & 7.46 & 3.83 & 3.95 \\
& MC & 59.57 & 51.65 & 21.77 & 22.46 \\
\hline \multirow{4}{*}{4} & CE & 2.73 & 1.76 & 0.78 & 0.75 \\
& GA & 15.23 & 11.87 & 3.47 & 3.46 \\
& PSO & 19.95 & 15.84 & 5.02 & 5.01 \\
& MC & 135.52 & 121.49 & 28.22 & 28.2 \\
\hline \multirow{4}{*}{5} & CE & 2.85 & 3.05 & 1.50 & 1.52 \\
& GA & 18.48 & 13.48 & 6.03 & 6.02 \\
& PSO & 24.56 & 19.56 & 8.69 & 8.7 \\
& MC & 156.1 & 159.42 & 48.71 & 48.96 \\
\hline \multirow{4}{*}{6} & CE & 5.83 & 4.08 & 2.04 & 2 \\
& GA & 26.78 & 23.31 & 7.34 & 7.26 \\
& PSO & 34.34 & 34.72 & 11.31 & 11.11 \\
& MC & 261.23 & 260.37 & 62.43 & 62.31 \\
\hline & & & & &
\end{tabular}

Comparing Scenario 1 with Scenario 2 and comparing Scenario 3 with Scenario 4, the simulation results are shown in Figures 2 and 3. From the indexes PSCWC and PRCAVT, the defense gets more combat benefits in Scenario 2 and Scenario 4 but spends more weapon costs. We can get the conclusion that the defense can save weapon costs to some extent by adjusting weight parameters $\alpha_{1}$ and $\alpha_{2}$ in different scenarios.

The computation time of solving the different scales DWTA problem is recorded in the four combat scenarios, and the relevant results are shown in Table 8; it is obvious that the index CT presents ascending sequence, that is, 
$\mathrm{CE}<\mathrm{GA} \approx \mathrm{PSO}<\mathrm{MC}$, and the computation cost of $\mathrm{CE}$ method is one-order-of-magnitude lower than that of GA and PSO method; consequently, we could get that the CE method has great advantage in solving speed over GA, PSO, and MC methods.

\section{Conclusion}

In this paper, a novel DWTA model under the resource requirement condition which is shoot-look-shoot variant is constructed, where weight parameters are introduced to balance the weapon costs and combat benefits. Subsequently, a hypothetical target is constructed to deal with the resource requirement condition, a new dynamic weapon target assignment method based on CE is introduced, and the detailed derivation process of using CE method to solve DWTA problem is given. Finally, to evaluate the solution quality of $\mathrm{CE}$ method, the dynamic weapon target assignment task based on CE, GA, PSO, and MC methods is simulated in several combat scenarios. Three conclusions are summarized based on the simulation results. Firstly, the expected combat purpose is achieved and a good supply of weapon costs are saved by dealing with the DWTA problem based on $\mathrm{CE}$ method under the condition that weapon inventory is adequate. Secondly, more combat benefits are acquired by dealing with the DWTA problem based on CE method under the condition that weapon inventory is inadequate. Thirdly, the solving speed of CE method is obviously faster than GA, PSO, and MC methods which more fit the real-time combat scenario. Besides, another strong point of the DWTA formulation is that adjusting weight parameters in different scenarios can save weapon costs to some extent.

For future research, we will focus on the promotion of CE method in its efficiency and time consumption by sample selection and appropriate parameter settings.

\section{Appendix}

Proof: Formulas (25) and (26) are equivalent.

Let $x_{i}(t)=j_{i}^{*}, j_{i}^{*} \in \widetilde{J}, \forall i \in W$, so $\prod_{i \in W} p\left(x_{i}(t) \mid i, t\right)=$ $p\left(j_{1}^{*} \mid i, t\right) \cdots p\left(j_{i}^{*} \mid i, t\right) \cdots p\left(j_{m}^{*} \mid i, t\right)$.

$$
\prod_{j \in J} p(j \mid i, t)^{g\left(x_{i}(t), j\right)}=p(1 \mid i, t)^{g\left(x_{i}(t), 1\right)} \cdots p\left(j_{i}^{*} \mid i, t\right)^{g\left(x_{i}(t), j_{i}^{*}\right)} \cdots p(n+1 \mid i, t)^{g\left(x_{i}(t), n+1\right)} .
$$

Because of $x_{i}(t)=j_{i}^{*}$, we can get $g\left(x_{i}(t), j_{i}^{*}\right)=1$ and $g\left(x_{i}(t), j\right)=0, \forall j \in \widetilde{J}$ and $j \neq j_{i}^{*}$.
Then, we can get the following formula: $\prod_{j \in \widetilde{J}} p(j \mid i, t)^{g\left(x_{i}(t), j\right)}=p\left(j_{i}^{*} \mid i, t\right)^{g\left(x_{i}(t), j_{i}^{*}\right)}=p\left(j_{i}^{*} \mid i, t\right)$.

Finally, we can get

$$
\begin{aligned}
\prod_{i \in W} \prod_{j \in J} p(j \mid i, t)^{g\left(x_{i}(t), j\right)} & =\prod_{i \in W} p\left(j_{i}^{*} \mid i, t\right)^{g\left(x_{i}(t), j_{i}^{*}\right)} \\
& =p\left(j_{1}^{*} \mid 1, t\right) \cdots p\left(j_{i}^{*} \mid i, t\right) \cdots p\left(j_{m}^{*} \mid m, t\right)=\prod_{i \in W} p\left(x_{i}(t) \mid i\right) .
\end{aligned}
$$

Consequently, formulas (25) and (26) are equivalent.

\section{Data Availability}

The simulation data used to support the findings of this study are included within the article.

\section{Conflicts of Interest}

The authors declare that they have no conflicts of interest.

\section{Acknowledgments}

This paper was funded by Science and Technology on Space Intelligent Control Laboratory for National Defense.

\section{References}

[1] A. S. Manne, "A target-assignment problem," Operations Research, vol. 6, no. 3, pp. 346-351, 1958.
[2] A. G. Kline, D. K. Ahner, and B. J. Lunday, "Real-time heuristic algorithms for the static weapon target assignment problem," Journal of Heuristics, vol. 25, no. 3, pp. 377-397, 2019.

[3] Y. L. Zhou, X. B. Li, Y. F. Zhu, and W. P. Wang, "A discrete particle swarm optimization algorithm applied in constrained static weapon-target assignment problem," in Proceedings of the World Congress on Intelligent Control and Automation (WCICA), pp. 3118-3123, Guilin, China, June 2016.

[4] C. M. Lai and T. H. Wu, "Simplified swarm optimization with initialization scheme for dynamic weapon-target assignment problem," Applied Soft Computing, vol. 82, 2019.

[5] T. Chang, D. Kong, N. Hao, K. Xu, and G. Yang, "Solving the dynamic weapon target assignment problem by an improved artificial bee colony algorithm with heuristic factor initialization," Applied Soft Computing, vol. 70, pp. 845-863, 2018.

[6] W. Xu, C. Chen, S. Ding, and P. M. Pardalos, "A bi-objective dynamic collaborative task assignment under uncertainty using modified MOEA/D with heuristic initialization," Expert Systems with Applications, vol. 140, 2020. 
[7] L. Mu, X. Qu, and P. Wang, "Application of sensor/weapontarget assignment based on multi-scale quantum harmonic oscillator algorithm," in Proceedings of the 2017 2nd International Conference on Image, Vision and Computing (ICIVC), pp. 1147-1151, Chengdu, China, June 2017.

[8] H. Chen, Z. Liu, Y. Sun, and Y. Li, "Particle swarm optimization based on genetic operators for sensor-weapon-target assignment," in Proceedings of the 2012 Fifth International Symposium on Computational Intelligence and Design, vol. 2, pp. 170-173, Hangzhou, China, October 2012.

[9] B. Xin, Y. Wang, and J. Chen, "An efficient marginal-returnbased constructive heuristic to solve the sensor-weapon-target assignment problem," IEEE Transactions on Systems, Man, and Cybernetics: Systems, vol. 49, no. 12, pp. 2536-2547, 2018.

[10] J. Li, J. Chen, and B. Xin, "Solving multi-objective multi-stage weapon target assignment problem via adaptive NSGAII and adaptive MOEA/D: a comparison study," in Proceedings of the 2015 IEEE Congress on Evolutionary Computation (CEC), pp. 3132-3139, Sendai, Japan, May 2015.

[11] C. Gao, Y. Kou, Y. Li, Z. Li, and A. Xu, "Multi-objective weapon target assignment based on D-NSGA-III-A," IEEE Access, vol. 7, pp. 50240-50254, 2019.

[12] L. Juan, C. Jie, and X. Bin, "Efficiently solving multi-objective dynamic weapon-target assignment problems by NSGA-II," in 2015 34th Chinese Control Conference (CCC), pp. 2556-2561, IEEE, Piscataway, NJ, USA, 2015.

[13] L. Yang, Z. Zhai, and Y. Li, "A multi-information particle swarm optimization algorithm for weapon target assignment of multiple kill vehicle," in Proceedings of the 2018 IEEE/ ASME International Conference on Advanced Intelligent Mechatronics (AIM), pp. 1160-1165, Auckland, New Zealand, July 2018.

[14] M. Z. Lee, "Constrained weapon-target assignment: enhanced very large scale neighborhood search algorithm," IEEE Transactions on Systems, Man, and Cybernetics-Part A: Systems and Humans, vol. 40, pp. 198-204, 2009.

[15] Y.-C. Liang and C.-Y. Chuang, "Variable neighborhood search for multi-objective resource allocation problems," Robotics and Computer-Integrated Manufacturing, vol. 29, no. 3, pp. 73-78, 2013.

[16] M. D. Rezende, B. S. L. P. De Lima, and S. Guimarães, “A greedy ant colony system for defensive resource assignment problems," Applied Artificial Intelligence, vol. 32, no. 2, pp. 138-152, 2018.

[17] X. Hu, P. Luo, X. Zhang, and J. Wang, "Improved ant colony optimization for weapon-target assignment," Mathematical Problems in Engineering, vol. 2018, Article ID 6481635, 14 pages, 2018.

[18] W. Duan, W. Yuan, and L. Pan, "Research on algorithm for dynamic weapon target assignment based on the improved Markov decision model," in 2018 37th Chinese Control Conference (CCC), pp. 8417-8424, IEEE, Piscataway, NJ, USA, 2018.

[19] T. Fu, Y. Liu, and J. Chen, "Improved genetic and ant colony optimization algorithm for regional air defense WTA problem," International Conference on Innovative Computing, vol. 1, pp. 226-229, 2006.

[20] B. Zhu, F. X. Zou, and J. H. Wei, "A novel approach to solving weapon-target assignment problem based on hybrid particle swarm optimization algorithm," in Proceedings of the International Conference on Electronic and Mechanical Engineering and Information Technology, pp. 1385-1387, Harbin, China, 2011.
[21] Y. Li and Y. Dong, "Weapon-target assignment based on simulated annealing and discrete particle swarm optimization in cooperative air combat," Acta Aeronautica Et Astronautica Sinica, vol. 31, pp. 626-631, 2010.

[22] R. Rubinstein, "The cross-entropy method for combinatorial and continuous optimization," Methodology and Computing in Applied Probability, vol. 1, no. 2, pp. 127-190, 1999.

[23] W. K. Saad, M. Ismail, R. Nordin, A. A. El-Saleh, and N. Ramli, "Multi-stage cross entropy optimization algorithm for hard combining schemes in cognitive radio network,", in Proceedings of the 2015 IEEE 12th Malaysia International Conference on Communications (MICC), pp. 113-118, Sarawak, Malaysia, November 2015.

[24] A. Becerra, J. I. de la Rosa, E. González, A. D. Pedroza, and N. I. Escalante, "Training deep neural networks with nonuniform frame-level cost function for automatic speech recognition," Multimedia Tools and Applications, vol. 77, no. 20, pp. 27231-27267, 2018.

[25] H. A. Le Thi, D. M. Nguyen, and T. Pham Dinh, "Globally solving a nonlinear UAV task assignment problem by stochastic and deterministic optimization approaches," Optimization Letters, vol. 6, no. 2, pp. 315-329, 2012.

[26] L. Huang, H. Qu, and L. Zuo, "Multi-type UAVs cooperative task allocation under resource constraints," IEEE Access, vol. 6, pp. 2169-3536, 2018.

[27] P. T. D. Boer, D. P. Kroese, and S. Mannor, "A tutorial on the cross-entropy method," Annals of Operations Research, vol. 134, pp. 19-67, 2005.

[28] A. Costa, O. D. Jones, and D. Kroese, "Convergence properties of the cross-entropy method for discrete optimization," Operations Research Letters, vol. 35, no. 5, pp. 573-580, 2007. 